A Freehand Graphic way of determining Stream Lines and Equipotentials

This content has been downloaded from IOPscience. Please scroll down to see the full text. 1907 Proc. Phys. Soc. London 2188

(http://iopscience.iop.org/1478-7814/21/1/306)

View the table of contents for this issue, or go to the journal homepage for more

Download details:

IP Address: 146.201.208.22

This content was downloaded on $03 / 10 / 2015$ at $02: 47$

Please note that terms and conditions apply. 
VT. A Freehand Graphic way of determining Stream Lines and Equipotentials. By L. F. Richardson *.

[Plate V.]

\section{SCheme of PaPer.}

I. Oy the need for new methods.

II. The first idea of freehand solution and confirmation of its aecuracy.

III. The conditions which the solution of $\nabla^{2} \mathrm{~V}=0$ must satisfy in order that it may be determinable by a single graph.

(a) When the guiding lines are normal to a family of surfaces. Possible types-test cases.

(b) Thin shells.

(c) Screw symmetry-example.

IV. Points and lines of equilibritum.

V. Equations other' than Laplace's-variable conductivity.

VI. Boundary conditions.

VII. Miscellaneous notes on draughtsmanship.

VIII. Estimation of errors.

\section{The Need for New Methods.}

THe Laplacian differential equation

$$
\frac{\partial^{2} V}{\partial x^{2}}+\frac{\partial^{2} V}{\partial y^{2}}+\frac{\partial^{2} V}{\partial z^{2}}=0
$$

has received an extraordinary amount of attention during the last century owing to the great number of plysical quantities, the space distribution of which can be determined from its integrals. The analytical integrals hitherto obtained by such means as Fourier series, Bessel functions, spherical and other luarmonics make it possible to determine the distribution when the bounclary conditions bear relation to certain simple types of surface, such as parallelipipeds, cylinders, spheres, ellipsoids, anchor rings, \&c.

Now for physical research this is well enough. It is usually possible to arrange the instruments so that the parts involved are of these simple forms. The wires may be wound in circular rings of small cross-section, as in Helmholtz's gralvanometer. The pieces of substance for' the measurement

* Iread Novewber 8, 1307. 
of specific properties may be shaped into square bars, as in Forbes's experiments on the flow of heat. Or, as in Kelvin's attracted disk electrometer, parallel plates may be made practically infinite by his device of the guard-ring.

But for the purposes of the engineer this is of very limited application. If he is to handle partial differential equations fieely, they must be applicable to bodies of most various shapes, such, for example, as the toothed core-plates of dynamos, the water surrounding ship shells and screw propellers, the space between turbine blades, and a host of other forms, too irregular to be readily described.

Further than this, the method of solution must be easier to become skilled in than are the usual methods with harmonic functions. Few have time to spend in learning their mysteries. And the results must be easy to verify-much easier than is the case with a complicated piece of algebra. Moreover, the time required to arrive at the desired result by analytical methods cannot be foreseen with any certainty. It may come out in a morning, it may be unfinished at the end of a month. It is no wonder that the practical engineer is shy of anything so risky.

Harmonic functions have, however, one very strong point in comparison to the methods put forward in this paper, and that is their accuracy. Once we have determined $V$ as an infinite series of harmonic functions, it is usually not much more labour to obtain an accuracy of 1 in a million than of 1 in ten.

Now it is true that in the determination of absolute electric standards measurements are made to 1 in 100,000 or to an even greater refinement. But for most chemical and physical work 1 in 1000 is more like the limit attained. And in any new branch of research, two, five, or even ten per cent. are very welcome. The root of the matter is that the greatest stimulus of scientific discovery are its practical applications. And here, in the design of machinery for example, cost rules everything, and this can seldom $b_{\ominus}$ foreseen as near as 1 per cent.

To sum up. The existing methods of solving Laplace's equation are susceptible of great accuracy, but they are slow and uncertain in time and, most serious of all, they can only 
be applied to very special boundary conditions. There is obviously a demand for a method of solving that group of partial differential equations - of which we may regard Laplace's as the simplest type-which shall, if necessary, sacrifice accuracy above 1 per cent., to rapidity, freedom from the danger of large blunders, and applicability to more various forms of boundary surface.

\section{The First Idea of Freehand Solution.}

The real simplicity of the space distributions of electric and magnetic phenomena,-so much disguised in the algebraic integrals of the differential equations, but rescued from confusion and clearly set forth by the vector analysts, Heaviside, Walker and others,- -leads one to hope for equally simple methods of calculating their numerical values with reference to any boundary whatever.

The beautiful figures of stream and equipotential surfaces published by Maxwell, Lamb and others as the result of harmonic analysis, and by Hele Shaw as the result of experiment, suggest that by imitating their characteristic properties freehand we may, in some small part, attain the result desired.

Maxwell in $\S 92$ of his 'Elementary Treatise on Electricity and Magnetism' speaks of tentative methods of altering known solutions of the Laplacian equation by drawing diagrams on paper and selecting the least improbable. The object of the present thesis is to point out that this method can do far more than merely alter known results, and that it may be so far from tentative as to yield an accuracy of one per cent of the range.

This method of treating potentials, although still far from combining all desirable qualities, and suffering from the restriction to certain types of symmetry, yet from its great freedom within those types may, it is hoped, supply to a certain extent the demand we have indicated.

On turning to Maxwell's figures and picking out those in which $V$ is independent of $z$ so that we have

$$
\frac{\partial^{2} V}{\partial w^{2}}+\frac{\partial^{2} V}{\partial y^{2}}=0
$$


OF DETERMINING STREAM IINES AND EQUIPOTENTIALS. 91

it will be seen that while the curves are of the most various shapes yet the chequerwork of all the diagrams possesses these two properties in common:-(1) the corners are orthogonal, (2) when the chequers are small enough the ratio of their length to breadth is the same in all parts of the field.

The proof of this follows most conveniently from the consideration of the motion of a liquid when the lines of flow lie in parallel planes and the motion is the same at all points of any normal to these planes. Draw three adjacent stream lines defining two adjacent tubes of flow.

Take two points $\mathrm{A}$ and $\mathrm{B}$ on the mid line of one tube, and from $A$ and $B$ draw normals to the direction of flow cutting the mid line of the other tube in $\mathrm{D}$ and $\mathrm{C}$ respectively.

Fig. 2.

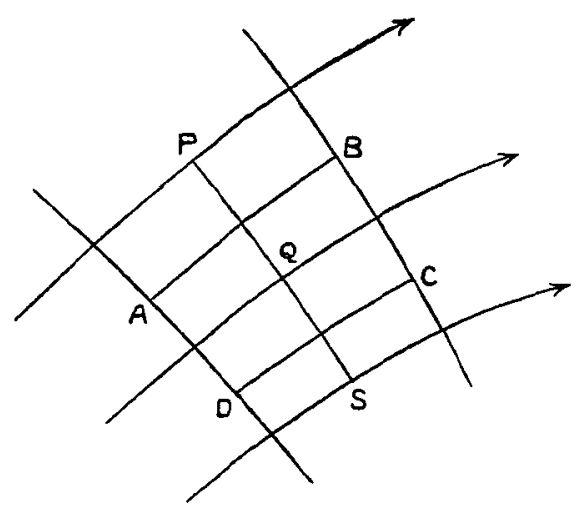

Halfway between $\mathrm{AD}$ and $\mathrm{BC}$ draw a line $\mathrm{PQS}$ normal to the direction of flow so that $\mathrm{PQ}$ is the width of one tube and QS of the other. Now if the fluid is incompressible and we have drawn the tubes so that the flow in each is the same, then the respective velocities are to one another inversely as $\mathrm{PQ}$ and $\mathrm{QS}$. Tet the velocities be $\frac{k}{\mathrm{PQ}}$ and $\frac{k}{\mathrm{QS}}$. Next let us take the line integral of the velocity round the small rectangle $A B C D$. The sides $A D$ and $C B$ are normal to the flow and so contribute nothing. The sides $A B$ and $C D$ 
contribute

$$
\mathrm{AB} \times \frac{k}{\mathrm{PQ}}-\frac{\mathrm{DC} \times k}{\mathrm{QS}}=k\left\{\frac{\mathrm{AB}}{\overline{\mathrm{PQ}}}-\frac{\mathrm{DC}}{\overline{Q S}}\right\} .
$$

Now $\frac{A B}{\overline{P Q}}$ is the ratio of the length along the flow to the breadth across the flow of the small chequer which bas $A, Q$, $B, P$, at the mid points of its four sides. It will be convenient to have a special name for this quantity, and I propose to call it the "chequer ratio" with the understanding that length along the flow is always in the numerator, and that the chequer is so small that its size no longer causes an appreciable deviation from the accuracy obtained by using infinitesimals. Then we have :-

Line integral of the velocity around $A B C D=k \times\left\{\begin{array}{l}\text { Difference between successive } \\ \text { chequer ratios in a direction } \\ \text { perpendicularly across flow. }\end{array}\right.$

Now the curl of the vector velocity is defined as the line integral round a small circuit divided by the area of that circuit-that is in this case by the area $A B C D$ which will in the limit be equal to the mean of the areas of the two adjacent chequers. So that we have :-

curl of the velocity $=k \frac{\begin{array}{c}\text { difference of successive chequer ratios in a directiou } \\ \text { perpendicular to velocity }\end{array}}{\text { mean chequer area }}$.

If the velocity has no curl the chequer ratio must not vary along any line normal to the flow. It may vary from one normal to another, but if on the other hand we prefer to make it constant all over the field, then at any point the distance between successive normals will be inversely as the flow, so that these normals will be contours drawn at equal intervals of a velocity potential.

To return : since the fluid is incompressible the condition for the existence of a stream function is satisfied, and since the stream-lines are drawn so that the flow between each successive pair is the same, it follows that these stream-lines are the contours drawn at equal intervals of a stream function $\psi$. Now it is proved in works on Hydrodynamics that $\frac{\partial^{2} \psi}{\partial x^{2}}+\frac{\partial^{2} \psi}{\partial y^{2}}$ is equal to the curl of the vector velocity. 
OF DETERMINING STREAM LINES AND EQUIPOTENTIALS. 93

Therefore :-

$\frac{\partial^{2} \psi}{\partial x^{2}}+\frac{\partial^{2} \psi}{\partial y^{2}}=k \frac{\begin{array}{c}\text { difference of successive chequer ratios in a direction } \\ \text { perpendicular to the contours of } \psi\end{array}}{\text { mean chequer area }}$.

And since $\psi$ may be any one-valued function of position on the plane, it is seen that all hydrokinetical considerations have been eliminated from the above equation, which is purely a proposition in differential geometry. The only implication being that contours are drawn at equal intervals of $\psi$ whatever be its physical meaning.

To draw chequers freehand so as to satisfy a difference relation of this sort between the chequer ratios is likely to be toilsome, and we will here consider only the case when $\nabla^{2} \psi=0$.

Fig. 2.

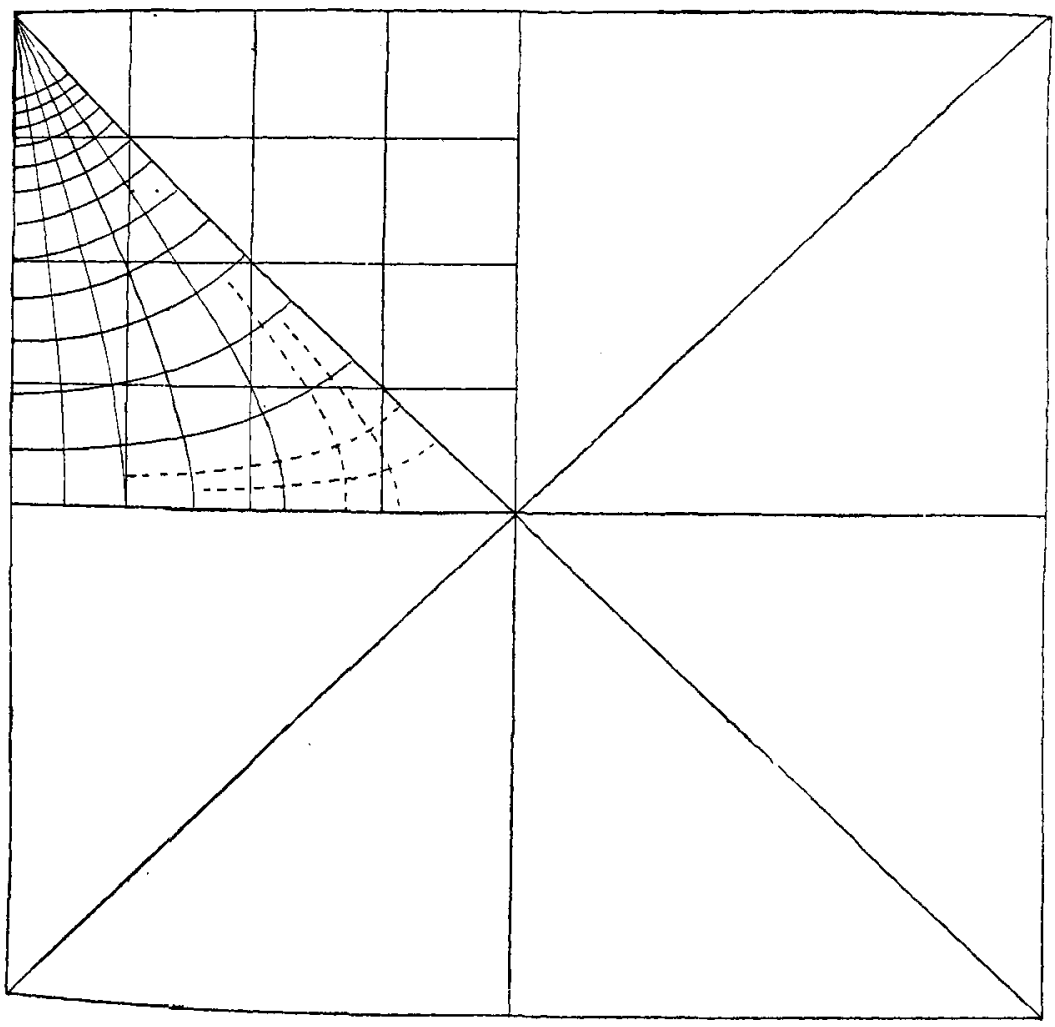


Supposing then that a chequerwork has been obtained in which the chequer ratio is everywhere the same and in which the given boundary conditions are satisfied, then by the uniqueness of the solution of $\frac{\partial^{2} V}{\partial c^{2}}+\frac{\partial^{2} V}{\partial y^{2}}=0$ this chequerwork gives us what we want.

It remains to be shown what accuracy may be expected from the freehand method. This is of course largely a personal matter. I exhibit my own handiwork. Others will doubtless obtain greater precision. Throughout this paper I have chosen test cases in which the analytical verification should not be too difficult. Hence the diagrams look rather stiff and formal and do not in any way do justice to the freedom of this graphic method.

Example of the solution of $\frac{\partial^{2} \mathrm{~V}}{\partial x^{2}}+\frac{\partial^{2} \mathrm{~V}}{\partial y^{2}}=0$.-Along one pair of opposite edges of a square $\mathrm{V}=1$, along the other pair $\mathrm{V}=0$. Find $\mathrm{V}$ at all points inside. By symmetry $V$ will be equal to $\frac{1}{2}$ along the diagonals. And again by symmetry, the lines joining mid-points of opposite sides will be normal to the contours of $V$. So that it is only necessary to find $V$ in half one-quarter of the square. Further, by symmetry, at a corner of the square the contours drawn at equal intervals of $V$ must make equal angles with one another. One starts then by ruling out an accurate square, putting in the diagonals, joining the mid-points of its sides and setting off the equal angles with a protractor. It is convenient to divide the range of $\mathrm{V}$ into ten equal parts. Having thus prepared the paper, lines were sketched and amended until further improvement became very slow. The pencil-lines were then firmly fixed in ink. Coordinate lines were drawn in and the values of $V$ at six points were read from the diagram and are given in parenthesis in the accompanying table. The whole work from the beginning of the drawing took two or three hours. 
OF DETERMINING STREAM LINES AND EQUIPOTENTIALS. 95

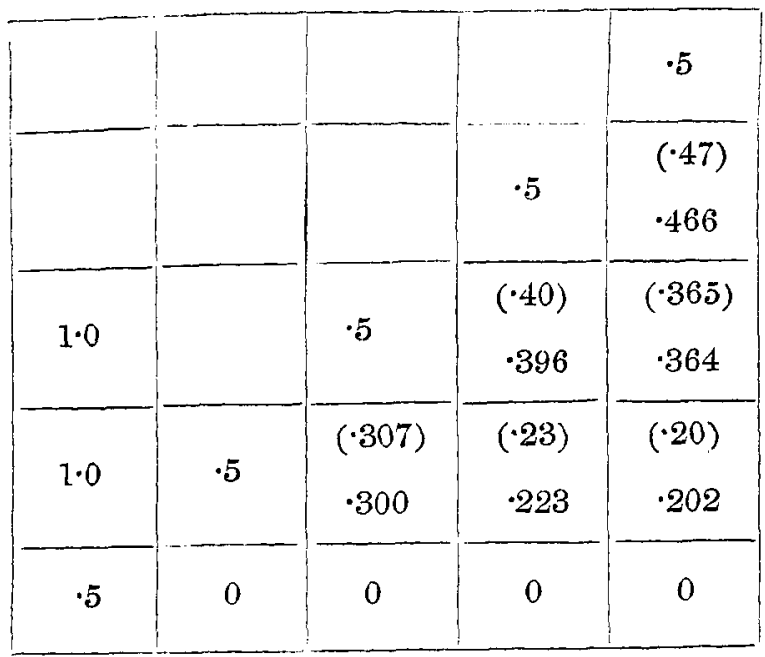

Not until this had been done did I look up the correct ralues which had been computed from the analytical solution

$$
\mathrm{V}=\frac{4}{\Pi} \sum_{m \text { odd }}(-1)^{\frac{m-1}{2}} \frac{1}{m} \operatorname{sech} \frac{m \Pi}{2} \cos m x \cdot \cosh m z \text {. }
$$

These are given in the table beside the numbers read from the graph. From these we find the errors $+\cdot 007,+\cdot 007$, $-.002,+.004,+\cdot 001,+\cdot 004$. Treating these as all of the same sign, their mean is 0042 .

The error of a graph may well be compared with the total range of $\mathrm{V}$ within which the determination was made freehand. In this case the range was 0.5 so that the mean error was 0.84 per cent. of the range, a degree of accuracy which would be sufficient for many purposes.

Having shown that the freehand method is a practical one in a plane, it will be well next to enquire to what types of symmetry it may be extended.

\section{Possible Types of Symmetry.}

Inasmuch as a single chequerwork is to determine the distribution in the whole of the space considered, we are confined to two coordinates. The freehand methorl at present offers 
nothing to compete with the analytic forms in which three coordinates appear, such as:

$$
\begin{aligned}
& \mathrm{V}=\sum_{m, n}\left(\mathrm{~A}_{m} \sin m x+\mathrm{B}_{m} \cos m u\right)\left(\mathrm{C}_{n} \sin n y+\mathrm{D}_{n} \cos n y\right)\left(\mathrm{F}^{-\sqrt{m^{2}+n^{2}}} \cdot z+\mathrm{G} e^{-}\right. \\
& \mathrm{V}=\sum_{m, k} e^{ \pm k z}\left(\mathrm{~A}_{m} \cos m \phi+\mathrm{B}_{n} \sin m \phi\right) J_{m}(k r),
\end{aligned}
$$

(Whittaker, 'Modern Analysis,' p. 318)

$\mathrm{V}=\sum_{n, m} \mathrm{C}_{m} r^{m}\left(\mathrm{~A}_{n} \cos n \phi+\mathrm{B}_{n} \sin n \phi\right)(\sin \theta)^{n} \cdot \frac{d^{n} \mathrm{P}_{m} \mu}{d \mu^{n}}$

or others like them.

$$
\text { (Byerly, 'Fourier's Series,' p. 196) }
$$

The expressibility of $\mathrm{V}$ in terms of two coordinates implies that $\mathrm{V}$ is constant along a certain family of lines in space, namely, the intersections of the surfaces over which the said two coordinates are respectively constant. Any particular type of symmetry is most conveniently distinguished by specifying the family of lines along each of which $\mathrm{V}$ must be constant.

As it will frequently be necessary to refer to these lines and to distinguish them from the normals to the surfaces $V=$ constant, I propose to call them the " guiding lines."

It is indeed conceivilble that by adding together several space distributions in each of which $\mathrm{V}$ is constant along a different family of lines, we might attempt the solution of problems which it may be impossible to treat by two coordinates directly, such for example as the motion of a perfect fluid past a three-bladed screw-propeller, or the electrostatic field due to a ring of electrons. It may even be possible to treat the most general distributions by means of sections of the potential surfaces drawn on the leaves of a book of tracingpaper. But these extensions must be left to those who desire the results. This paper deals only with two coordinates.

Case (a). The guiding lines are everywhere orthogonal to a family of surfaces.-Let these be the surfaces over each of which $\gamma=\mathrm{F}_{3}(x, y, z)$ is constant.

Then choosing a particular surface, say $\gamma=\gamma_{0}$, we wish to draw thereon a chequerwork of orthogonal lines, and we wish this chequerwork, by the motion of each point of it along the guiding line at that point, to sweep out two families of surfaces 
in space, in sucb a way that one family may be equipotentials and the other stream-surfaces. This requires that these two families, which we may denote by

$$
\alpha=\mathrm{F}_{1}(x, y, z)=\text { const., } \quad \beta=\mathrm{F}_{3}(x, y, z)=\text { const. },
$$

should be everywhere orthogonal. Therefore the surfaces $\alpha, \beta, \gamma$ are mutual orthogonal, and consequently the surfaces $\gamma$ must satisfy the condition necessary in any member of a triply orthogonal system (Salmon, ' Geometry of 'Three Dimensions,' 4 th ed. $\$ \S 476$ to 486$)$.

But more than this. For we wish to be unrestricted as to the direction of the orthogonal traces of $\alpha$ and $\beta$ drawn upon the surfaces $\gamma$. Therefore, since three mutually orthogonal surfaces necessarily intersect in their lines of curvature (loc. cit. $\$ 304$ ), it follows that at every point of the surfaces $\gamma$ there are lines of curvature in every direction. The only form which possesses this property is the sphere or its limit the plane. Therefore the surfaces $y$ are either spheres or planes. This is necessary. We have not proved that it is sufficient. As frequent reference will be made to the theorem.s proved in Lamé's Leçons sur les coordonnées curvilignes, it will be convenient to employ expressions such as (Lamé, $\S x i .15$ ) to indicate equation 15 of $\$ \mathrm{xi}$. of this treatise. The relation of our notation to Iuamé's is that his $\rho \rho_{1} \rho_{2}$ are replaced by $\alpha \beta \gamma$ and that $\alpha \beta \gamma$ are used as subscripts respectively instead of absence of subscript, 1 and 2 . Otherwise the notations are the same.

In particular, if $F$ is any function of position, we will denote by $\mathrm{II}_{F}$ the quantity

$$
\frac{1}{\sqrt{\left(\frac{\partial F}{\partial x}\right)^{2}+\left(\begin{array}{l}
\partial \mathrm{F} \\
\partial y
\end{array}\right)^{2}+\left(\frac{\partial \mathrm{F}}{\partial z}\right)^{2}}}
$$

which is the reciprocal of the space-rate of $F$ along the normal to the surface $\mathrm{F}=$ constant.

Gnsider the lamina bunded by the two spheres $\gamma$ and $\gamma+\delta \gamma$. The thickness of the lamina is $\mathrm{H}_{\gamma} \delta \gamma$. If $\alpha=\mathrm{V}=$ the potential, then the surfaces $\beta=$ constant are surfaces of flow. Denoting in like muner the distances between two adjacent surfaces of these families by $\mathrm{H}_{a} \delta \%, \mathrm{H}_{\beta} \delta \beta$, we see that $\mathrm{H}_{a} \delta$ ce YOL. XXr. 
and $\mathrm{H}_{\beta} \delta \beta$ are the length and breadth of a chequer traced on the surface $\gamma$.

A tube of flow is bounded by the four surfaces $\beta, \beta+\delta \beta$, $\gamma, \gamma+\delta \gamma$. And its cross section is therefore $\mathrm{H}_{\beta} \cdot \mathrm{H}_{\gamma} . \delta \beta . \delta \gamma$.

Now if the flux has no divergence, then along a tube of flow magnitude of flux multiplied by cross-section $=$ constant. But the magnitude of $f l u x$ is equal to the negative space-rate of the potential $\alpha$ along the line of flow, and this is $-\frac{1}{H_{a}}$. Therefore along a line of flow $\frac{\mathrm{H}_{\beta} \cdot \mathrm{H}_{\gamma}}{\mathrm{H}_{a}} . \delta \beta . \delta \gamma$ must be constant in order that the vector space-rate of the scalar $\alpha$-the Hamiltonian $\nabla \alpha$-shall have no divergence. Or equivalently the condition that

$$
\nabla^{2} \alpha=0 \text { is } \frac{\partial}{\partial \alpha}\left(\frac{\mathrm{H}_{\beta} \cdot \mathrm{H}_{\gamma}}{\mathrm{H}_{\alpha}}\right)=0 .
$$

Now we may by freehand trial and amendment so arrange the orthogonal lines on the surface $\gamma_{0}$ that the above relation shall hold true on the surface $\gamma_{0}$; but we must further enquire what conditions the spheres $\gamma$ must satisfy in order that $\frac{\partial}{\partial \alpha}\left(\frac{\mathrm{H}_{\beta} \cdot \mathrm{H}_{\gamma}}{\mathrm{H}_{\alpha}}\right)=0$ shall be true for all values of $\gamma$ when it is true for one $\gamma_{0}$; and this moreover when $\alpha$ and $\beta$ are otherwise undetermined.

At this stage the fact that the surfaces $\gamma$ are spheres makes a remarkable simplification. For supposing for a moment that they did not possess this property and that $\gamma_{\gamma^{\prime}}^{\alpha}$ and $\gamma_{\gamma}^{\beta}$ were their principal radii of curvature at any point, then by (Lamé, § xxx. 24)

$$
\frac{1}{r_{\gamma}^{\alpha}}=\frac{h_{\gamma}}{h_{\alpha}} \cdot \frac{\partial h_{\alpha}}{\partial \gamma}, \quad \frac{1}{r_{\gamma}^{\beta}}=\frac{h_{\gamma}}{h_{\beta}} \cdot \frac{\partial h_{\beta}}{\partial \gamma}
$$

where $h_{\alpha}=\frac{1}{\mathrm{H}_{\alpha}}$, and similarly for $\beta$ and $\gamma$.

Equating the two curvatures,

Now

$$
h_{\beta} \cdot \frac{\partial h_{\alpha}}{\partial \gamma}=h_{\alpha} \frac{\partial h_{\beta}}{\partial \gamma}
$$

$$
\frac{\partial}{\partial \gamma}\left(\begin{array}{c}
\mathrm{H}_{\beta} \\
\tilde{\mathrm{H}}_{\alpha}^{-}
\end{array}\right)=\frac{\partial}{\partial \gamma}\left(\frac{h_{a}}{h_{\beta}}\right)=\frac{-h_{\alpha} \frac{\partial h_{\beta}}{\partial \gamma}+h_{\beta} \frac{\partial h_{\alpha}}{\partial \gamma}}{h_{\beta}^{2}}
$$


OF DETRRMINING STREAM LINES AND EQUIPOTENTIALS. 99

and therefore vanishes; so that the chequer rutio $\frac{H_{a}}{\bar{H}_{\beta}}$ is a function of $\alpha$ and $\beta$ only.

As has already been stated, to make $\nabla^{2} \alpha=0$ on the surface $\gamma_{0}$ we must have

$$
\frac{\partial}{\partial \alpha}\left(\frac{\mathrm{H}_{\beta} \mathrm{H}_{\gamma_{0}}}{\mathrm{H}_{\alpha}}\right)=0
$$

which may conveniently be arranged by making

$$
\frac{\mathrm{H}_{\alpha}}{\mathrm{H}_{\beta}}=k_{0} \mathrm{H}_{\gamma_{0}}
$$

where $k_{0}$ is a constant, so that the chequer ratio is given as a function of position on $\gamma_{0}$. This is more than sufficient in that it makes

$$
\frac{\partial}{\partial \beta}\left(\frac{\mathrm{H}_{\beta} \mathrm{H}_{\gamma_{0}}}{\mathrm{H}_{\alpha}}\right)
$$

vanish as well, but the loss of generality involved is found not to matter, while the simplicity gained is a great convenience. Next, because $\frac{H_{\alpha}}{\mathrm{H}_{\beta}}$ is independent of $\gamma$ it follows that on any other surface $\gamma_{1}$ we still have

$$
\frac{\mathrm{H}_{\alpha}}{\tilde{\mathrm{H}}_{\beta}}=k_{0} \mathrm{H}_{\gamma_{0}} \text {. }
$$

l3ut if $\nabla^{2} \alpha=0$ is to be satisfied on this second surface we must there have

$$
\frac{\mathrm{H}_{\alpha}}{\overline{\mathrm{H}}_{\beta}}=k_{1} \mathrm{H}_{\gamma},
$$

where $k_{1}$ is a second constant. Therefore regarding $\gamma_{0}$ as fixed and $\gamma_{1}=\gamma$ as movable we have $\mathrm{H}_{\gamma}=\mathrm{H}_{\gamma_{0}} \times$ a function of $\gamma$ only.

But $\mathrm{H}_{\gamma_{0}}$ is a function of $\alpha$ and $\beta$ only.

This relation is equivalently expressed by the two equations

$$
\frac{\partial^{2}}{\partial \gamma \partial \alpha}\left(\log \mathrm{H}_{\gamma}\right)=0, \quad \frac{\partial^{2}}{\partial \gamma \partial \beta}\left(\log \mathrm{H}_{\gamma}\right)=0,
$$

whence by (Lamé, $\S \mathrm{xxx} .24$ )

$$
\frac{\partial}{\partial \gamma}\left(\frac{1}{h_{\alpha} r_{\alpha}^{\gamma}}\right)=0, \quad \frac{\partial}{\partial \gamma}\left(\frac{1}{h_{\beta} r_{\beta}^{\gamma}}\right)=0 \text {. }
$$

But if the surfaces $\gamma$ are planes, then by the equations 
(Lamé, $\S \mathrm{xxx}, 24$ ) already quoted

$$
\frac{\partial}{\partial \gamma}\left(\frac{1}{h_{\alpha}}\right)=0=\frac{\partial}{\partial \gamma}\left(\frac{1}{h_{\beta}}\right)
$$

and consequently $\frac{1}{r_{\alpha}^{\gamma}}$ and $\frac{1}{r_{\beta}^{\gamma}}$ are independent of $\gamma$. But it is shown by Lamé ( $\$$ xxxviii.) that the curvature of the arc of intersection of the surfaces $\alpha$ and $\beta$ is equal to

$$
\sqrt{\left(\frac{1}{r_{a}^{\gamma}}\right)^{2}+\left(\frac{1}{r, \gamma}\right)^{2}}
$$

So that if the radius of curvature of this arc be $p$ then $p$ is independent of $\gamma$. But $p$ is equal to the length of the normal from the point considered onto the line of ultimate intersection of two consecutive planes of the family $\gamma$ which pass one on either side of the point considered. As the plane moves this length must remain constant. And as this is to be true for every point in space, it is easy to see that if the surfaces $\gamma$ are planes they must intersect in a common axis. We have in this case symmetry about an axis. $O r$ if the axis be at an infinite distance, the planes are parallel, and we have $V$ independent of one of the Cartesian coordinates $x, y, z$. But if, guite generally, the surfaces $\gamma$ are spheres we have only $\frac{l_{a}}{l_{\beta}}$ independent of $\gamma$ and therefore $\frac{r_{\alpha}^{\gamma}}{r_{\beta}^{\gamma}}$ independent of $\gamma$. If the centres of the spheres $\gamma$ lie in a straight line, then since the orthogonal traces of the surfaces $\alpha$ and $\beta$ on a splere $\gamma$ may turn round anyhow, we may choose for $\beta$ the planes intersecting in the line of the centres of the spheres $\gamma$. Then $r_{\beta}^{\gamma}=0$, and consequently $r_{\alpha}^{\gamma}$ is independent of $\gamma$ so that the trices of $a=$ const. on the planes $\beta$ are circles. This is the system of toroidal coordinates which has been treated by Professor Hicks in Phil. Trans. 1881, Part II. Now the above reasoning would lead us to expect in these a type of symmetry which can be dealt with by two coordinates-other than symmetry about an axis. But on referring to Hicks's formulæ it is easy to show that this is not possible, for if $\mathrm{V}$ be made indeyendent of either of those two of his coordinates which determine position in a plane passing through the axis, then the other of these two will not divide out of the equation $\nabla^{2} V=0$, so that all three coordinates 
must still be present in the integrals. Clearly then, our deductions, though necessary, are not sufficient. I have little doubt that the omission lies in this : that to leave us unrestricted as to the direction of the orthogonal traces of $\alpha$ and $\beta$ upon the surfaces $\gamma$, it is not sufficient that the surfaces $\gamma$ should be spheres. For the curves normal to $\gamma$ which we have called the "guiding lines" must be such that they form one set of lines of curvature of any surface whaterer passing through them. To satisfy this condition it seems likely that except when the radius of the spheres $\gamma$ is infinite, the guiding lines will have to be straight and the spheres concentric. This is the symmetry when $V$ is independent of the ralius in spherical coordinates, but may vary anyhow with the latitude and longitude.

The only case remaining uninvestigated is that in which the surfaces $\gamma$ are spheres with centres which do not lie on a straight line.

By this application of Lamés formula, aided by those due to Hicks, we have discovered no new type of symmetry which allows two coordinates to be used instead of three. We have proved that within the stated limits the wellknown types are the only possible ones. A summary of these may be useful.

Summary of Types of Symmetry when the gniding lines are orthogonal to a family of surfices.

\begin{tabular}{|c|c|c|c|}
\hline Guiding lines. & Chequer ratio. & $\begin{array}{l}\text { If } \nabla^{2} V \text { is made equal } \\
\text { to } f(V, \alpha, \beta) \text { over one } \\
\text { surface } \gamma_{0} \text { its value } \\
\text { on the others will be }\end{array}$ & $\begin{array}{l}\text { Analytical } \\
\text { metiods. }\end{array}$ \\
\hline Parallel straights. & Coustant. & $\nabla^{2} V=f(V, \alpha, \beta)$. & $\begin{array}{l}\text { Conijugate } \\
\text { functions. }\end{array}$ \\
\hline $\begin{array}{l}\text { Circles with their } \\
\text { centres on a com- } \\
\text { mon axis and } \\
\text { their planes nor- } \\
\text { mal thereto. }\end{array}$ & $\begin{array}{l}\text { Proportional to } \\
\text { distanee } \\
\text { frum axis. }\end{array}$ & $\nabla^{2} \nabla=f(\nabla, \alpha, \beta)$. & $\begin{array}{c}\text { Zonal harinouics } \\
\text { of the } \\
\text { cylindrical, } \\
\text { spherical, } \\
\text { spheroidal, } \\
\text { and toroidal } \\
\text { spstems. }\end{array}$ \\
\hline $\begin{array}{l}\text { Radii from a com- } \\
\text { mon point. }\end{array}$ & Constant. & $\begin{array}{l}\nabla^{2} \nabla=\frac{f(\nabla, a, \beta)}{\gamma^{2}} \\
\text { where } r \text { is the distance } \\
\text { tron the radiant point. }\end{array}$ & \\
\hline
\end{tabular}


Eirample of Symmetry about an A.xis.-Byerly in his 'Fourier's Series and Spherical Harmonics,' p. 230, sets the following problem :- "A cylinder of radius one metre and altitude one metre has its upper surface kept at temperature $100^{\circ}$, and its base and convex surface at the temperature $15^{\circ}$, until the stationary temperature is set up. Find the temperature at points on the axis $25 \mathrm{~cm} ., 50 \mathrm{~cm}$, and $75 \mathrm{~cm}$. from the base. and also at a point $25 \mathrm{~cm}$. from the base and $50 \mathrm{~cm}$. from the axis." To solve this the first thing necessary is to prepare a chart bearing chequers of the appropriate shape for each distance from the axis. The graph of any solution of $\nabla^{2} \mathrm{~V}=0$ symmetrical about an axis would serve this purpose. For example several of the figures out of Muxwell's 'Electricity and Mignetism ' would do. But I preferred to prepare a standard chart by ruling equidistant parallel equipotentials normal to the axis of revolution, and then stream-lines parallel to the axis at distances from it proportional to the square roots of the natural numbers $0,1,2,3,4,5$, \&c. The cross section of the cylindrical shell enclosed between successive stream-lines is then the sime for every pair, and the chequer ratio proportional to the distance from the axis. This having been done in red ink, a sheet of tracing-paper was pinned over it, the section of our given cylinder was drawn in black and equipotentials and lines of flow were drawn in pencil. These were then rubbed out and amended with the aim of making the pencil chequers everywhere very similar to the red rectangles underneath. When improvement became slow, the blurred lines were made firm and definite with ink and the chequers considered individually and marked as to whether they were too square or too thin. The lines were then drawn on a clean sheet of tracing-paper, the chequers again examined individually, and tinally the lines fixed in ink (see fig. 3). Coordinate lines were then ruled and the values of $V$ at their intersections were read from the graph. This process, from the ruling in of the given contour to the determination of $\mathrm{V}$ in numbers, took me four hours. The analytical method would perbaps have been more rapid in this case; but for an irregular shaped contour with an irregular boundary distribution the freehand solution would still take about the same 
OF DETERMINING STREAM LINES AND EQUIPOTENTIALS. 103 time, while analytical methods may be almost indefinitely tedious.

Fig. 3.

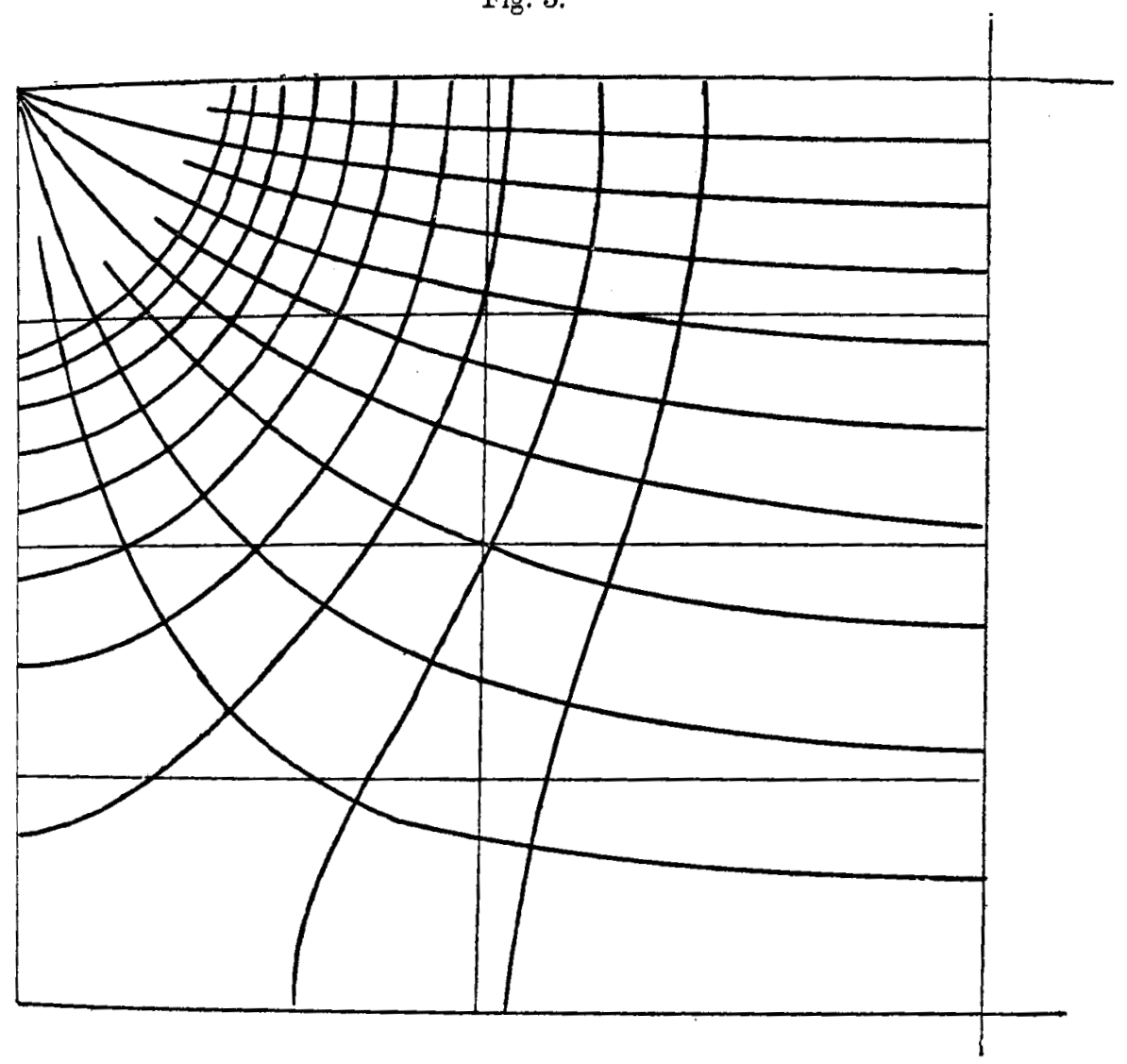

The results are tabulated below :-

Distance from base of cylinder $\ldots . .25 \quad 50 \quad 75 \quad 25$ cms.

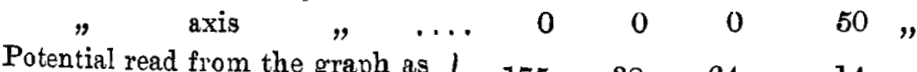

$\begin{array}{lllll}\begin{array}{l}\text { Potential read from the graph as } \\ \text { decimal of unit range. }\end{array} & \cdot 175 & \cdot 38 & \cdot 64 & \cdot 14\end{array}$

$\left.\begin{array}{c}\text { Last multiplied by the actual range } \\ \left(100^{\circ}-15^{\circ}\right)\end{array}\right\} \quad \begin{array}{llll}14 \cdot 9 & 32 \cdot 3 & 54 \cdot 4 & 11 \cdot 9\end{array}$

Last $+15^{\circ}=$ actual temperature $\quad . .29 \cdot 9 \quad 47 \cdot 3 \quad 69 \cdot 4 \quad 26 \cdot 9$

Not until these numbers had been written clown did I look 
at the correct ralnes found by Byerly from the Bessel function scries, namely :-

$\begin{array}{lrrrr} & 29 \cdot 6 & 47 \cdot 6 & 71 \cdot 2 & 25 \cdot 8 \\ \text { Errors of freehand determination. } & +\cdot 3 & -.3 & -.8 & +1 \cdot 1\end{array}$

Disregarding signs the mean of these errors is 63 which is equal to 0.74 per cent. of the range of $85^{\circ}$.

Example of the type of symmetry when $\mathrm{V}$ is independent of the radius in spherical coordinates. - "On a uniform spherical shell there are equal sources at the north and south poles and equal sinks at the extremities of a diameter lying in the equatorial plane. The sources and sinks send out and receive uniformly in all directions. The flux has no divergence except at the sources and sinks and no curl anywhere. Find the distribution of potential on the surface." Todo this we might draw orthogonal lines on the surface of a globe so as to make the chequers ratio constant. Or because, in Mercator's projection, any small part on the globe transforms into a small part of the same shape on the map, we may transform the boundary conditions and obtain the required solution by drawing chequers of constant chequer ratio on the map. Blank Mercator projections suitable for this work may be obtained from George Philip \& Son, Fleet Street. In the present example the lines of flow radiating from the pole become lines straight, parallel and equidistant at infinity. And as the graph progressed it was found that by their symmetry with the sinks on the equator, the foregoing condition must be very nearly satisfied at $10^{\circ}$ from the poles, a region which is within the confines of the map. Again, in this case it is only necessary to determine $\mathrm{V}$ in one octant of the sphere, and symmetry helps us in other ways. The accompranying graph (fig. 4) is the best of four or five separate attempts. The time taken to make these was collectively four hours. Special attention was given to the equipotential curve which passes mid-way between the two equatorial sinks, and as the result of the aforesaid trials it was found to pass through a point $44^{\circ}$ due north of the sink on the equator. This suggested that the true value should be $45^{\circ}$, and on looking at a sphere this is seen to follow from symmetry althongh it was not obvious on the map. Thus again we have a confirmation of 
OF DETERMINING STREAM IINES AND EQUIPOTENTIALS. 105 the passable accuracy of the graphic method-the error here is 1 degree in 90 or 1.1 per cent. of the range.

So far we have only treated the problem as relating to a spherical shell. But we may next suppose the sphere solid

Fig. 4.

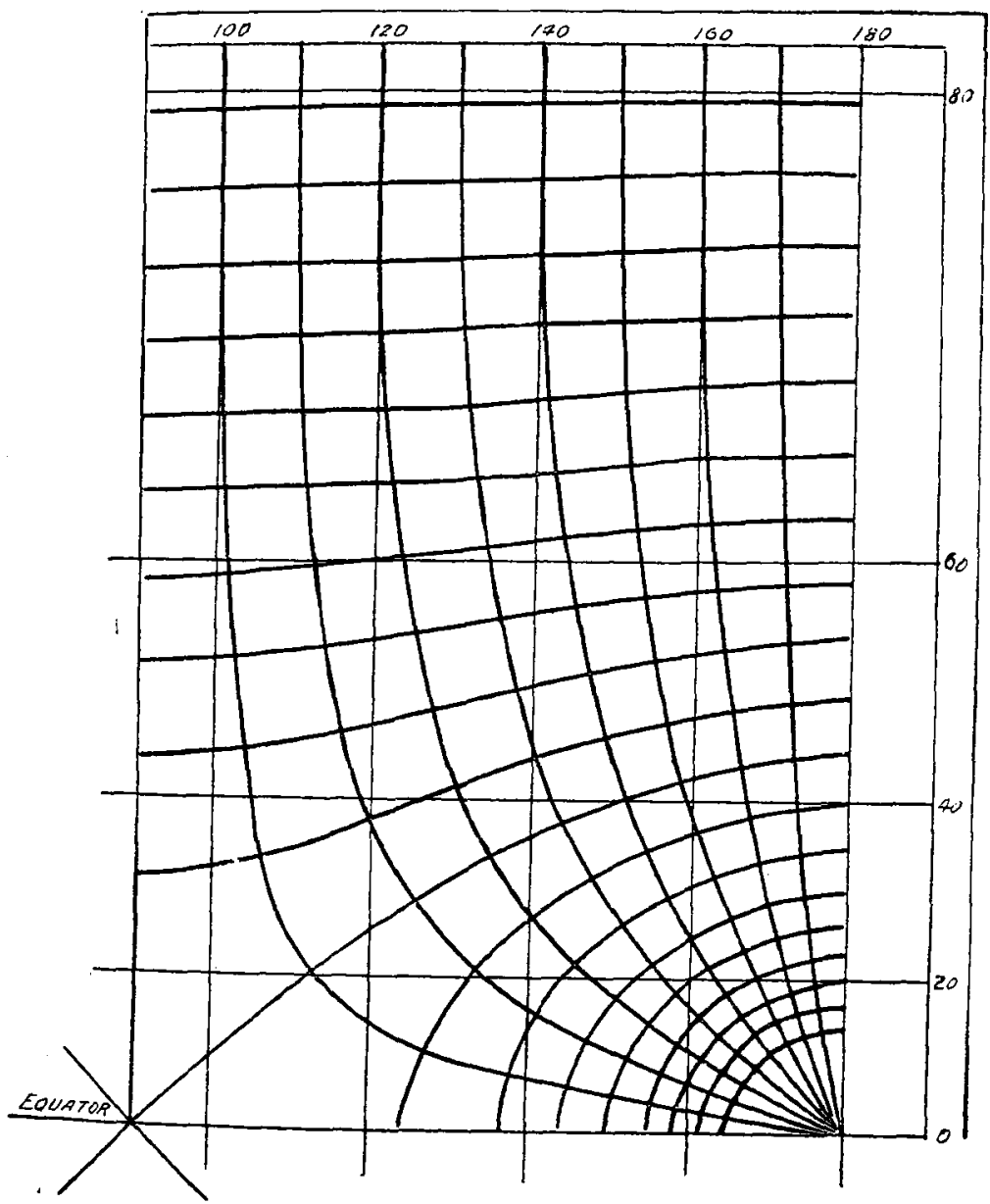

and $V$ to be independent of the radius. We will then have a solution of Laplace's equation in space. Since the chequer ratio is constant, the magnitude of the flox is inversely proportional to the linear dimensions of the chequer (on the 
sphere not on the map) and is consequently proportional to $\frac{1}{r}$ along any guiding line, $r$ being the radius. But if we draw any small cone enclosing the polar axis-iwhich is now a line source-we see that the outflow between two spheres $r$ and $r+\delta r$ is proportional to $\delta r \times$ (magnitude of flux) $\times$ (perimeter of the trace of the cone), and by the above this is pro. portional simply to $\delta r$. Therefore the polar axis must be a line source of uniform strength and similarly for any other source or sink when the guiding lines are straights passing through a common point-the strength must be independent of the radius.

Case (b).-General method for conduction in a thin shell of any shape, the thickness and conductivity being any given functions of position on its surface, and all conditions being constant throughout the thickness of the shell at any point of its surface. Take a solid bounded by a surface of the shape of the shell and draw small rectangles at numerous points of the surface, so that their chequer ratio $=\frac{\text { length along flow }}{\text { breadth across flow }}$ is directly proportional to the product of the thickness and conductivity at each point of the shell. For then the flow through each chequer will be the same. Suppose that these standard chequers are in some distinctive colour, say red. Now lay off in black the boundary conditions of the special problem and draw a black chequerwork to have the same chequer ratio as the red at each point, much as was done for symmetry about an axis. The standard red chequers need not be connected so as to form two systems of orthogonal lines but may be scattered anyhow over the surface, all that is necessary is that they should be sufficiently small and numerous.

Or it may be convenient to use a projection of the surface as was done in the case of the spherical shell above.

Case (c).-When there exists no family of surfaces normal to the guiding lines. Without pausing for generalities wo will proceed at once to:-

Screw symmetry alout an axis.-Let us discuss this with the aid of cyliudrical coordinates $r, \phi, z$. At a point $\mathrm{P}$ on 
the axis $O Z$ let a perpendicular be drawn extending to infinity. This perpendicular, which is to project only on one side of the axis, is imagined to revolve round the axis and slide along the same with proportional velocities. In one rotation round the axis let it move $l$ along the axis. Then the line sweeps out a surface, at all points of which the expression $z-\frac{l}{2 \pi} \phi$ is constant. Let us put $z-\frac{l}{2 \pi} \phi=\omega$.

Then as $\omega$ varies we pass from one of these screw surfaces to another formed by shifting the first parallel to $z$. The range of the coordinate $\boldsymbol{\omega}$ is from 0 to $l$. The intersections of $\omega=$ const. with the cylinders $r=$ const. are a family of screw-threads.

Let $d \eta$ be an element of distance measured along any sorew guiding line, so that $\frac{d \eta}{d z}=\frac{\text { length of turn of screw }}{l}=$ a function of $r$ only. And let us make $V$ a line:ır function of distance along each surew-thread so that $\frac{\partial V}{\partial \eta}=$ a function
of $r$ only.

Then as $d \eta, d \omega$, and $d r$ are in perpendicular directions they are independent and

$$
\frac{\partial V}{\partial z}=\frac{\partial V}{\partial \omega} \cdot \frac{\partial \omega}{\partial z}+\frac{\partial V}{\partial \eta} \cdot \frac{\partial \eta}{\partial z}+\frac{\partial V}{\partial r} \cdot \frac{\partial r}{\partial z}
$$

which reduces to $\frac{\partial V}{\partial z}=\frac{\partial V}{\partial \omega}+$ a function of $r$ only.

Therefore

Again,

$$
\begin{aligned}
& \frac{\partial^{2} V}{\partial z^{2}}=\frac{\partial}{\partial z}\left(\frac{\partial V}{\partial \omega}\right)=\left(\begin{array}{l}
\partial \omega \\
\partial z \cdot \frac{\partial}{\partial \omega}+\frac{\partial \eta}{\partial z} \cdot \partial \\
\partial \eta
\end{array}\right) \frac{\partial V}{\partial \omega} \\
& =\frac{\partial^{2} V}{\partial \omega^{2}}+\frac{\partial \eta}{\partial z} \cdot \frac{\partial}{\partial \omega}\left(\frac{\partial V}{\partial \eta}\right)=\frac{\partial^{2} V}{\partial \omega^{2}} .
\end{aligned}
$$

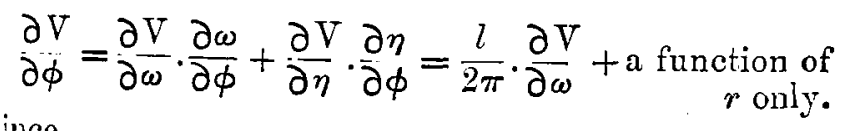

Since

$$
\frac{d \eta}{d \phi}=\frac{\text { length of turn of screw }}{2 \pi}=\text { a function of } r \text { only. }
$$


And :o

$$
\begin{aligned}
\frac{\partial^{2} V}{\partial \phi^{2}}=\frac{l}{2 \pi} \cdot \frac{\partial}{\partial \phi}\left(\frac{\partial V}{\partial \omega}\right)=\frac{l}{2 \pi}\left(\begin{array}{ll}
\partial \omega & \frac{\partial}{\partial \phi} \cdot \frac{\partial \omega}{\partial \omega}
\end{array}\right. & \left.+\frac{\partial \eta}{\partial \phi} \cdot \frac{\partial}{\partial \eta}\right) \frac{\partial V}{\partial \omega} \\
& =+\frac{l^{2}}{4 \pi^{2}} \cdot \frac{\partial^{2} V}{\partial \omega^{2}}
\end{aligned}
$$

Now substitute these values of $\frac{\partial^{2} V}{\partial z^{2}}$ and $\frac{\partial^{2} V}{\partial \phi^{2}}$ in the ex. pression for $\nabla^{2} V$ in cylindrical coordinates, and we have

$$
\nabla^{2} V=\frac{\partial^{2} V}{\partial r^{2}}+\frac{1}{r} \frac{\partial V}{\partial r}+\left(1+\frac{\gamma^{2}}{1 \pi^{2} r^{2}}\right) \frac{\partial^{2} V}{\partial \omega^{2}}
$$

which contains only two coordinates $r$ and $\omega$. So that if we make $\nabla^{2} V=f(V, r, \omega)$ over any surface the same will be true throughout the whole region filled by the screw-threads passing through the surface, provided that $\frac{\partial V}{\partial \eta}$ is such as to make $\frac{\partial^{2} V}{\partial r^{2}}+\frac{1}{r} \frac{\partial V}{\partial r}$ constant along every guiding screw. One way of satisfying this is to make $V$ increase by the same amount per turn of the screw, along every screw-thread and so that $\frac{\partial^{2} V}{\partial r^{2}}$ and $\frac{\partial V}{\partial r}$ are both constant along every guiding screw.

In the following pages, except where specially indicated, we will consider only the case $\frac{\partial V}{\partial \eta}=0$.

As this result does not appear to be given in the textbooks, it may be well to confirm it by a slightly different line of reasoning, as follows. The tangent of the angle between the tangent to any screw-threads and a plane normal to the axis of symmetry is $\frac{l}{2 \pi r^{\prime}}$.

Therefore the first space rate of any function of position $V$ aloug the tangent to a screw-thread is

$$
\frac{1}{\sqrt{l^{2}+4 \pi^{2} r^{2}}}\left\{2 \pi r \frac{\partial}{r \partial \phi}+l \frac{\partial}{\partial z}\right\} \mathrm{V} \text {. }
$$

If $\frac{\partial V}{\partial \eta}=$ a function of $r$ only, along every screw-thread then we have a function of $r$ only $+2 \pi \frac{\partial V}{\partial \phi}=-l \frac{\partial V}{\partial z}$ 
throughont the whole region. Therefore this last equation will still remain true after differentiation by $\phi$ or by $z$, thus

$$
\begin{gathered}
-\frac{2 \pi}{l} \frac{\partial^{2} V}{\partial \phi^{2}}=\frac{\partial^{2} V}{\partial \bar{\phi} \partial z}, \\
\frac{\partial^{2} V}{\partial \phi \partial z}=-\frac{l}{2 \pi} \partial^{2} V \\
\frac{\partial}{2} z^{2}
\end{gathered}
$$

Equating the two values of $\frac{\partial^{2} V}{\partial \phi \partial z}$ thus oltained we have

$$
\frac{\partial^{2} V}{\partial \phi^{2}}=\frac{l^{2}}{4 \pi^{2}} \frac{\partial^{2} V}{\partial z^{2}}
$$

which on substitution in the expression for $\nabla^{2} V$ gives

$$
\nabla^{2} \mathrm{~V}=\frac{\partial^{2} \mathrm{~V}}{\partial r^{2}}+\frac{1}{r} \frac{\partial \mathrm{V}}{\partial r^{2}}+\left(1+\frac{l^{2}}{4 \pi^{2} r^{2}}\right) \frac{\partial^{2} \mathrm{~V}}{\partial z^{2}}
$$

But it is now to be observed that if the distribution of $\mathrm{V}$ on any plane passing through the axis of symmetry is known, then $\mathrm{V}$ is determined ererywhere. And on such a fixed plane the contours of $z$ are identical with those of $\omega$. So that we may replace $z$ ly $\omega$ in the last equation, and the previous result is confirmed.

We have shown that if we make $\nabla^{2} V=f(\mathrm{~V}, \boldsymbol{r}, \boldsymbol{\omega})$ over any surface intersecting all the screw-threads, the same will be true throughout the whole region, with the stated provisions as to the value of $\frac{\partial V}{\partial \eta}$. The geometrical meaning of this result is that if we draw any infinitesimal rectangle normal to one of the screw-threads and draw screw-threads through each of its four comers, then the infinitesimal tube thus formed will be everywhere rectangular in normal cross section, and more than this, the rectangle will have the sane ratio of length to brealth and will he of the same size at all points along the tube. Iror if we consider one pair of opposite faces of the tube as equipotentials and the other pair as lines of flow, then these properties are seen to follow from the fact that $\nabla^{2} \mathrm{~V}$ is constant along a screw-thread when $\mathrm{V}$ is constant along the same. And indeed these properties are inmediately obrious from the appearance of the system. 
Consequently, if we take any family of surfaces $\alpha$ passing through the guiding screw-threads, there will always be an orthogonal family of surfaces $\beta$, also passing through the screw-threads. If the surfaces $\alpha$ are the contours drawn at equal intervals of the potential $\mathrm{V}$ the surfaces $\beta$ are streamsurfaces. And $\frac{\mathrm{H}_{\alpha}}{\mathrm{H}_{\beta}}$ may be named the "Chequer Ratio" consistently with what has gone before. If $\nabla^{2} \alpha$ is to vanish we must have

$$
\frac{\partial}{\partial \alpha}\left(\frac{\mathrm{H}_{a}}{\mathrm{H}_{\beta} \times \frac{(\text { length of portions of successive screw-threads intercepted }}{\text { between two stream-lines lying on the same stream-surface })}}\right)=0 \text {. }
$$

Since the screw system is uniform the length of the portions of successive screw-threads intercepted between two stream-lines lying on the same surface $\beta$ can be proportional to nothing else than the length of one turn of the screwthread at the radius considered. For the two stream-lines in question must by symmetry make equal angles with planes normal to the axis of the screw, at each pair of points lying on the same screw-thread. So that the projection of the distance between the said pair of points onto the axis of the screw will be always the same fraction of $l$ as the points move from one screw-thread to another.

Now the length of an arc $d s$ of a screw-thread being

$$
\sqrt{d z^{2}+r^{2} d \theta^{2}}=d \theta \sqrt{\frac{l^{2}}{4 \pi^{2}}+r^{2}},
$$

the length of one turn is

$$
\int_{\theta=0}^{\theta=2 \pi} d s=2 \pi \sqrt{\frac{l^{2}}{4 \pi^{2}}+r^{2}}=l \sqrt{1+\frac{4 \pi^{2} r^{2}}{l^{2}}}
$$

Therefore we may satisfy the equation $\nabla^{2} \alpha=0$ by making $\frac{\mathrm{H}_{\alpha}}{\mathrm{H}_{\beta}}$ proportional to $\sqrt{1+\frac{4 \pi^{2} r^{2}}{\iota^{2}}}$ as the radius varies. A table giving the values of $\sqrt{1+\frac{4 \pi^{2} r^{2}}{l^{2}}}$ for a number of values of $\frac{r}{l}$ is annexed. 
OF DETERMINING STREAM LINES AND EQLIPOTENTIALS. 111

\begin{tabular}{|c|c|c|c|c|}
\hline \multirow{2}{*}{$\frac{r}{i}}$. & \multirow{2}{*}{$\begin{array}{l}\sqrt{1+\frac{4 \pi^{2} \gamma^{2}}{l^{2}}} \\
l \text { times this equals aro } \\
\text { of one turn of threacl. }\end{array}$} & \multicolumn{2}{|c|}{ For standard chart. } & \multirow{2}{*}{$\begin{array}{l}\frac{l}{2 \pi r} \\
\text { equals tangent of angle } \\
\text { between helix and circle. }\end{array}$} \\
\hline & & $\mathrm{H}_{a^{*}}$ & $\mathbf{H}_{\beta}$. & \\
\hline $\begin{array}{l}0 \\
.05 \\
.10 \\
.15 \\
.20\end{array}$ & $\begin{array}{l}1 \cdot 0000 \\
1 \cdot 0482 \\
1 \cdot 1811 \\
1 \cdot 3741 \\
1 \cdot 6060\end{array}$ & $\begin{array}{l}.5507 \\
.5639 \\
.5985 \\
.6456 \\
.6979\end{array}$ & $\begin{array}{l}1 \cdot 8157 \\
1.7735 \\
1.6707 \\
1.5410 \\
1.4328\end{array}$ & $\begin{array}{l}\infty \\
3.1831 \\
1.5916 \\
1.0610 \\
.7958\end{array}$ \\
\hline $\begin{array}{r}.30 \\
.40 \\
.50 \\
60 \\
70 \\
80 \\
.90 \\
1.00\end{array}$ & $\begin{array}{l}2 \cdot 1338 \\
2 \cdot 7049 \\
3 \cdot 2969 \\
3 \cdot 9003 \\
4 \cdot 510.5 \\
5 \cdot 1251 \\
5 \cdot 7426 \\
6.3623\end{array}$ & $\begin{array}{r}.8045 \\
.9058 \\
1 \cdot 0000 \\
1.0877 \\
1.1697 \\
1.2468 \\
\cdot 3198 \\
1.3892\end{array}$ & $\begin{array}{r}1 \cdot 2430 \\
1 \cdot 1040 \\
1.0000 \\
.9194 \\
.8550 \\
.8021 \\
.7577 \\
.7199\end{array}$ & $\begin{array}{l}.53059 \\
.39789 \\
.31831 \\
.26526 \\
.22736 \\
.19894 \\
.17684 \\
.15916\end{array}$ \\
\hline $\begin{array}{l}1.5 \\
2.0 \\
2.5 \\
3.0 \\
3.5\end{array}$ & $\begin{array}{r}9.478 \\
12.606 \\
15.740 \\
18.876 \\
22.014\end{array}$ & $\begin{array}{l}1 \cdot 6955 \\
1 \cdot 9554 \\
2 \cdot 1850 \\
2 \cdot 3028 \\
2 \cdot 5840\end{array}$ & $\begin{array}{l}.5808 \\
.5114 \\
.4577 \\
.4179 \\
.3870\end{array}$ & $\begin{array}{l}\cdot 10610 \\
.07958 \\
.06366 \\
.05305 \\
.04547\end{array}$ \\
\hline$\infty$ & $\infty$ & $\infty$ & 0 & $\infty$ \\
\hline
\end{tabular}

It is hoped that these values are correct to less than half a unit in the last place.

As there is no surface normal to the screw-threads, it is not possible to draw standard rectangles of the appropriate chequer ratio for each distance from the axis. But as the whole distribution of $\mathrm{V}$ is determined when the section of it by a plane passing through the axis of the screw is known, we may draw on this plane the sections of tubes formed by the surfaces $\alpha$ and $\beta$ in such a way that $\frac{\mathrm{H}_{\alpha}}{\overline{\mathrm{H}}_{\beta}}$ is proportional to

$$
\sqrt{1+\frac{4 \pi^{2} r^{2}}{l^{2}}}
$$

The sections of these tubes will in general not be rectangles; in fact, the angles and ratio of sides of the chequers formed by the traces of $\alpha$ and $\beta$ on the plane $\phi=$ constant will both now depend on the orientation of the chequer as well as on its distance from the axis. It will therefore be necessary 
to make a chart of stanclard chequers in rarious orientations at a number of distances from the axis. Plate $V$. is such a chart. The rectangles in the right-hand margin represent normal cross sections of the tubes formed by the surfaces $\alpha$ and $\beta$. In a line with each of these are five sections of a tuhe of the same size and shape by the plane of the paper, when the angle between one face of the tube and the normal to the axis of the screw is sncessively $0^{\circ}, 22 \frac{1}{2}^{\circ}, 45^{\circ}, 67 \frac{10}{2}$, $90^{\circ}$. In order to be clearly visible these parallelograms are drawn quite large. What each rolly represents is the shape of an infinitesimal chequer situated at the central point of the large one. Practically the difference will not be important.

Now this standard diagram can be covered by a sheet of tracing-paper, and two intersecting families of lines drawn on the tracing-paper in such a way that the parallelograms formed by them are everywhere similar to the chequers underneath, which have the same distance from the axis and the same orientation on the paper. Then if this tracing-paper plane rotate round the axis and slide along it so as to follow the guiding lines, the equipotential lines on the paper will sweep out the contours at equal intervals of $V$ in space in such a way that $\nabla^{2} V=0$ and the other family of lines will sweep out stream-surfaces.

A quantity which it is frequently necessary to determine is the magnitude of the flux

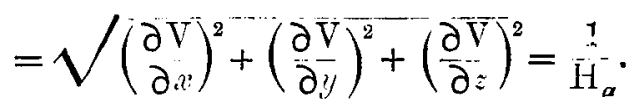

Since

$$
\begin{aligned}
& \mathrm{H}_{a} \\
& \tilde{\mathrm{H}}_{\beta}^{-}
\end{aligned}=\mathrm{A} \mathcal{N} / \frac{4 \pi^{2}{ }^{2}}{l^{2}}
$$

where $A$ is an absolute constant, we must have

$$
\frac{1}{\mathrm{H}_{\alpha}}=\mathrm{A} \frac{\sqrt{1+\frac{4 \pi^{2} r^{2}}{l^{2}}-}}{\mathrm{H}_{\beta}}
$$

So that $\beta$ is a stream function analogous to the forms in $n$ s $\theta$ when the guirling lines are parallel straights or circles with their centres on, and their planes normal to, a common axis. 
OF DETERMINING STREAM LINES AND EQUIPOTENTIALS. 113

In trpes previously studied, when the graph was drawn on a surface normal to the guiding lines, $\mathrm{H}_{\boldsymbol{\alpha}}$ and $\mathrm{H}_{\boldsymbol{\beta}}$ were proportional to the length and breadth of a chequer and could be measured directly. But here we must first compare the linear dimensions of a freehand chequer with those of the standard oblique section of the tube bounded by two stream-surfaces and two equipotentials, and then refer to the normal section of the same tube in the right-hand margin of the chart.

The standard chequers were obtained in the following manner :-

$\frac{\mathrm{H}_{a}}{\mathrm{H}_{\beta}}$ being equal to constant $\times \sqrt{1+\frac{4 \pi^{2} r^{2}}{l^{2}}}$, some other relation is necessary to determine $\mathrm{H}_{\alpha}$ and $\mathrm{H}_{\beta}$ separately. The relation $\mathrm{H}_{a} \times \mathrm{H}_{\beta}=1$ was chosen for this purpose, as this gives a neat appearance to the standard chart. It was also found convenient to make the constant such that $\frac{\mathrm{H}_{\beta}}{\mathrm{H}_{\alpha}}=1$ when $\frac{r}{l}=0.5$. The values of $\mathrm{H}_{\alpha}$ and $\mathrm{H}_{\beta}$ were calculated and are given in the accompanying table. The sides of the rectangles in the right-hand margin of the standard chart were drawn proportional to $2 \mathrm{H}_{\alpha}$ and $2 \mathrm{H}_{\beta}$.

To obtain the slant section, the tangent of the angle between the tangent a guiding-line and the plane normal to the axis of the screw, was first calculated. It is equal to $\frac{l}{2 \pi r}$, and is given in the table under that head. The rectangles were then projected with ruler and compasses in a manner which is perhaps sufficiently indicated by fig. 5 , which shows the construction when $\frac{r}{l}=0.05$ and the angle between a radius from the axis of symmetry and the tangent plane to the surface $\alpha=$ constant meeting at the point considered is $45^{\circ}$.

Of the innumerable solutions of $\nabla^{2} \mathrm{~V}=0$ possessing screw Symmetry of the sort described, which may be obtained by the aid of this standard chart, perhaps the simplest is the field due to a belical line source, such for example as the distribution of temperature in a mass of electrically insulating 
Fig. 5.

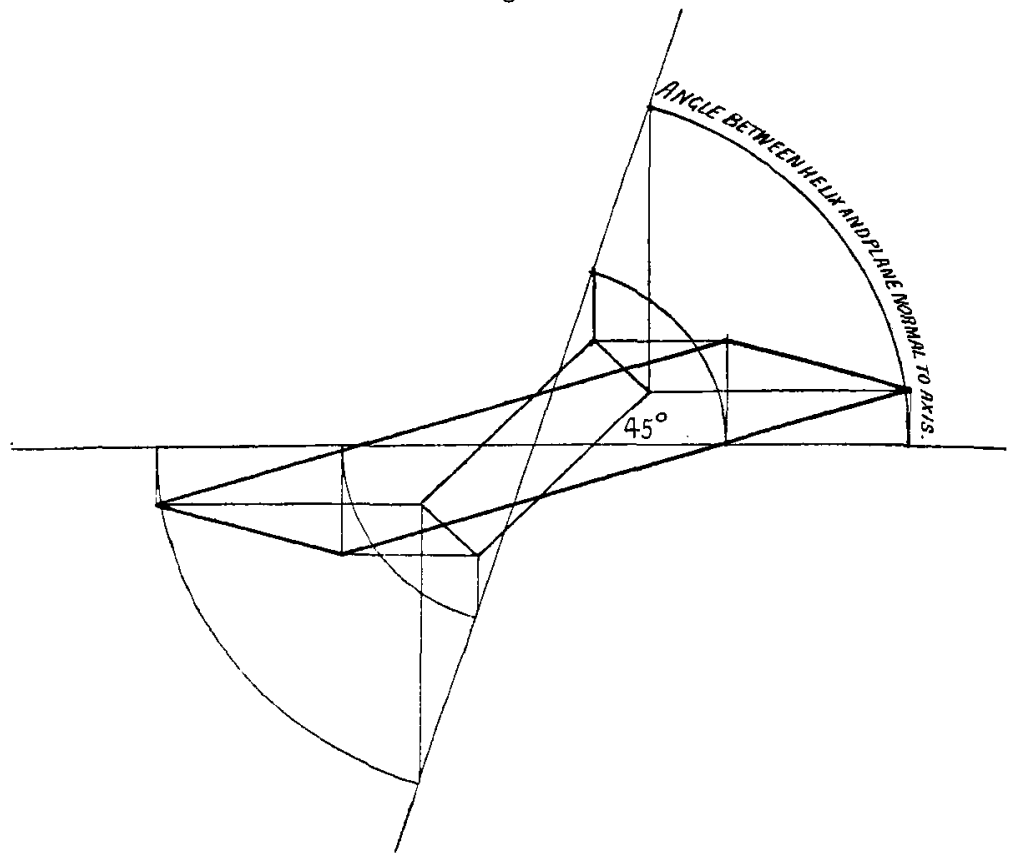

material which encloses a helical copper wire carrying an electric current. To avoid the introduction of a difficulty not characteristic of screw symmetry, I have assumed a core of non-conducting material in the form of a circular cylinder surrounding the axis. This relieves us of the necessity of considering the axial line of equilibrium, which would otherwise have to be treated by an extension of the method in Section IV. The external surface of the medium is also taken as a circular cylinder and is assumed to be at constant temperature. Consistently with our boundary conditions we may suppose that $\frac{d V}{d \eta}=0$. Now symmetry will help us in several ways, for since the chequer ratio on the standard chart is the same whether any particular half-turn of the screw passes over or under the chart, one sees on beginning to make the drawing, that the two surfaces $\omega=$ const., which pass respectively through the electric current and half-way between two adjacent turns of the current, must be surfaces 
OF DETERMINING STREAM LINES AND EQULPOTENTIALS. 115

of flow. Again, very close to the electric current the flow of heat will be nearly the same as that due to a straight current tangential to the helix, that is to say, the lines of flow will be normals to the helix and the isothermals will approximate to circular cylinders concentric about the tangent.

The particular dimensions chosen were $\stackrel{r}{l}=0.05$ for the core, 0.3 for the source, and 0.5 for the outer cylinder.

Fig. 6.

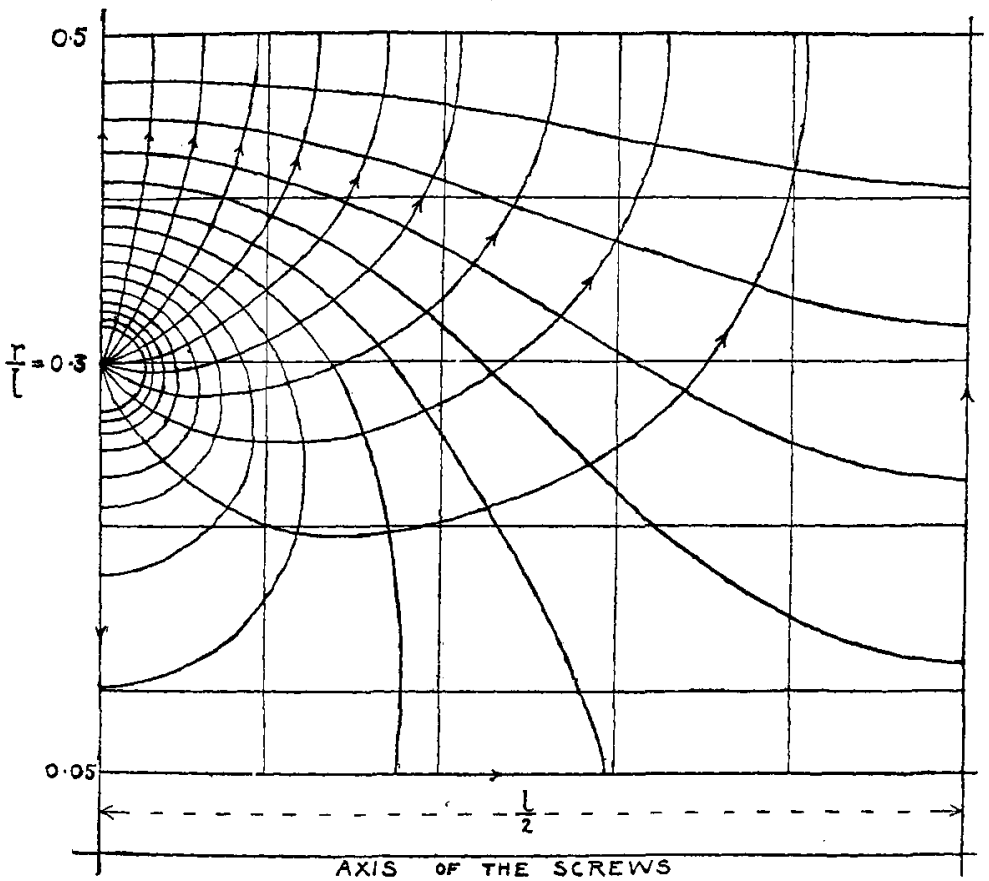

Owing to orientation of the chequer affecting its shape this graph took twice or thrice as long to adjust as did the others in this paper. Its errors are discussed in Section VIII. hereafter.

The magnetic field due to the helical current may doubtless be determined in a very similar manner. Here $\frac{\partial V}{\partial \eta}$ will be a constant other than zero and the cyclical properties of the field will add a further complication. 
IV.

We have hitherto passed over without mention the peculiarities relating to points of equilibrium-these are points at which the first space-rate of the potential vanishes in all directions. In the neigbbourhood of these the chequers become unusually large, and if any chequer goes right up to an equilibrium point it will not have the shape characteristic of its neighbours, but will take a peculiar form of its own.

There are several diagrams of this in Maxwell's 'Electricity and Magnetism.' See, for example, vol. ii. fig. xvii.

Now if $\mathrm{V}$ be expressed in terms of rectangular coordinates $x$ and $v$ lying in the plane of the graph with their origin at the equilibrium point, then linear terms in $V$ must vanish, and we have

$$
\mathrm{V}=\mathrm{A} u^{2}+\mathrm{B} \omega+\mathrm{Co}^{2}+\mathrm{E} u^{3}+\mathrm{F} u^{2} v+\mathrm{G} u v^{2}+\text { terms of higher }
$$

Now let us make $\nabla^{2} \nabla$ vanish.

For guiding lines parallel straight and normal to the plane of the graph

$\nabla^{2} \nabla=\frac{\partial^{2} V}{\partial u^{2}}+\frac{\partial^{2} V}{\partial v^{2}}=2(\mathrm{~A}+\mathrm{C})+u(6 \mathrm{E}+2 \mathrm{G})+v(2 \mathrm{~F}+6 \mathrm{H})$.

When the graph is on a plane passing through an axis about which there is symmetry of revolution and $u$ is normal to this axis, we must add to the above value of $\nabla^{2} \mathrm{~V}$ the term

$$
\frac{1}{r} \frac{\partial \mathrm{V}}{\partial u}=2 \frac{u}{r} \mathrm{~A}+\frac{v}{r} \mathrm{~B}+3 \frac{u^{2}}{r} \mathrm{E}
$$

where $r$ is distance from the axis.

Now when the point considered is not on or close to the axis, it will be possible to put in so many chequers that the first two chequers in any direction from the equilibrium point require for their measurement so small a range of $u$ and $v$ that the fractions $\frac{u}{r}, \frac{v}{r}, \& c$. , will be small, and therefore the additional terms which come in for symmetry about an axis may be neglected, and we have the same form for $\nabla^{2} V$ in both cases. 
Further, since Mercator's projection does not alter the shape of any small pieces, $\nabla^{2} \mathrm{~V}$ will have the same form in the neighbourhood of an equilibrium point on the Mercator's plan of the distribution on a sphere.

This being so, the general form of $\mathrm{V}$ in all three cases is $\mathrm{V}=a\left(u^{2}-v^{2}\right)+b . u v+g\left(u^{3}-3 u v^{2}\right)+h\left(v^{3}-3 u^{2} v\right)+$ higher terms where $a, b, g$, and $h$ are arbitrary constants. When the ratios of $a$ and $b$ to the succeeding coefficients do not vanish, then the first two terms are all that we need consider. Now it may easily be shown that by a proper rotation of the axes of reference, so that $u v$ tranform to $u_{1} v_{1}$, the sum of these two terms may be transformed into either of them separately We need therefore only consider one, say $b u_{1} v_{1}$. The contours of this function are hyperbolas and are orthogonal to those of $a\left(u_{1}{ }^{2}-v_{1}{ }^{2}\right)$. The ratio $\frac{a}{b}$ is determined by the chequer ratio in the neighbourhood of the equilibrium point.

A graph of this function for the special case of unit chequer ratio is given in Webster's 'Dynamics,' p. 525, and shows that two equipotentials meet at right angles at the equilibrium point, and that two stream-lines also pass through the same point and bisect the angles between the equipotentials. The eight curved chequers which meet in the point each have consequently three comers of $90^{\circ}$ and one of $45^{\circ}$. A graph of this function may be used as a "standard equilibrium point" to keep the eye informed of the necessary proportions of the first and second ring of chequers surrounding the point.

If, however, the coefficients $a$ and $b$ vanish, while $g$ and $h$ do not, then the terms of the 3rd degree become all important.

By rotating the axes the sum of the two terms of the $3 \mathrm{rd}$ degree may be reduced to either separately. A rough graph of the contours of these functions is given by Fiske in Merriman \& Woodward's 'Higher Mathematics,' p. 248. Here three equipotentials intersect in the equilibrium point. And three stream-lines bisect the angles of $60^{\circ}$ which are formed in this way. 
Now when a graph has to be drawn and is found to contain an equilibrium point, the general arrangement of the potential will give us the clne as to whether two, three, or more equipotentials intersect in the equilibrium point. And this being known, we have only to draw in the standard type at the proper dimensions and chequer ratio.

When the graph is drawn on a plane passing through an axis about which there is screw symmetry of the sort described in Section III $c$, then the appearance is different, for we have to add to the value of $\nabla^{2} \mathrm{~V}$ for circular symmetry about an axis the term

$$
\frac{l^{2}}{4 \pi^{2} r^{2}} \cdot \frac{\partial^{2} \mathrm{~V}}{\partial v^{2}}=\frac{l^{2}}{4 \pi^{2} r^{2}}(2 \mathrm{C}+2 u \mathrm{G}+6 v \mathrm{H}) .
$$

And therefore writing

we have

$$
1+\frac{l^{2}}{4 \pi^{2} r^{2}}=\mathrm{R}^{2}
$$

$$
\mathrm{V}=a\left(\mathrm{R}^{2} u^{2}-v^{2}\right)+b \cdot u v+g\left(\mathrm{R}^{2} u^{3}-3 u v^{2}\right)+h\left(v^{3}-3 \mathrm{R}^{2} u^{2} v\right) .
$$

A simpler way of looking at the matter is to consider a tiny plane element normal to the guiding screw which forms the line of equilibrium. The normals to the surfaces $\omega=$ const. lie in this plane. If $d \mathrm{~S}_{\omega}$ distance along such a normal, then

$$
d \omega=d \mathrm{~S}_{\omega} \sqrt{\left(\frac{\partial \omega}{\partial z}\right)^{2}+\left(\frac{1}{r} \frac{\partial \omega}{\partial \phi}\right)^{2}}=d \mathrm{~S}_{\omega} \sqrt{1+\frac{l^{2}}{4 \pi^{2} r^{2}}} .
$$

Substituting this in the expression of $\nabla^{2} V$ in terms of $r$ and $\omega$ we have

$$
\nabla^{2} V=\frac{\partial^{2} \mathrm{~V}}{\partial r^{2}}+\frac{1}{r} \frac{\partial \mathrm{V}}{\partial r}+\frac{\partial^{2} \mathrm{~V}}{\partial \mathrm{S}_{\omega}{ }^{2}}
$$

just as if $\mathrm{S}_{\omega}$ was $z$ in circular symmetry about an axis. From this we see that the appearance of the equilibrium point on a small plane element normal to the guiding screw will be exactly similar to the forms already dealt with. Its appearance on a plane which passes through the axis of the screw may be sketched without much difficulty by comparing the chequers in the right-hand margin of the standard chart with their projections as drawn in the middle of the chart. 


\section{Equations other than Laplace's.}

It has been shown above that in order to solve the equation

$$
\frac{\partial^{2} \mathrm{~V}}{\partial x^{2}}+\frac{\partial^{2} \mathrm{~V}}{\partial y^{2}}=\text { any given function of } \mathrm{V}, x, y \text {, }
$$

a relation between differences of chequer ratios has to be satisfied. And the same will be found to be true for the other forms of the equation $\nabla^{2} \mathrm{~V}=\mathrm{a}$ function (of $\mathrm{V}$ and of position) which can be treated by two coordinates. A difference relation of the sort referred to would involve the comparison of each chequer with a standard set having: graded chequer ratios, followed by the calculation of $\nabla^{2} \nabla$ by arithmetic. And although it would doubtless be possible to carry out the necessary operations, yet it would almost certainly be quicker and more accurate to use arithmetical finite differences altogether, writing in the numerical values of $\mathrm{V}$ at a set of points on the paper and adjusting theso numbers. until the finite difference equation is satisfied,-in a manner which may be described in a future paper. In view of this I will not attempt to elaborate freehaud methods for $\nabla^{2} V=$ a given function of $\mathrm{V}$ and of position.

There are, however, certain common space distributions which may be treated graphically with simplicity although they do not satisfy $\nabla^{2} V=0$.

Firstly, when the conductivity is a continuous function of position, and the direction of the flux is normal to the contours of a potential, and the magnitude of the flux is the maximum space-rate of the potential multiplied by the conductivity, and the flux has no divergence. For example : the flow of heat and electricity in isotropic but non-homogeneous bodies, or the soakage of water in a saturated subsoil the upper layers of which are more porous than those below. Let $\mathrm{K}$ be the conductivity and suppose that it is constant along each guiding line but varies from one such line to another. Then, when the lines have a family of surfaces normal to them we must have

$$
\frac{\partial}{\partial \alpha}\left(\frac{\mathrm{H}_{\beta} \cdot \mathrm{H}_{\gamma} \cdot \mathrm{K}}{\mathrm{H}_{\alpha}}\right)=0
$$


in order that the flux shall not diverge. This is very easily assured by preparing the paper with standard chequers having their chequer ratio $\frac{\mathrm{H}_{\alpha}}{\mathrm{H}_{\beta}}$ proportional to $\mathrm{H}_{\gamma} \cdot \mathrm{K}$. In fact, we have an example of this in Section III $a$ above; for circular symmetry about an axis may be regarded for this purpose as flow between parallel planes in a medium having conductivity directly proportional to the distance from the axis. And reciprocally.

Similarly in the case of screw symmetry, standard chequers are to be prepared having

$$
\frac{\mathrm{H}_{a}}{\mathrm{H}_{\beta}} \text { proportional to } \mathrm{K} \sqrt{1+\frac{4 \pi^{2} r^{2}}{l^{2}}} \text {. }
$$

Two other cases can probably be treated freehand, namely, the flow of heat in bodies where the conductivity varies with the temperature, and, of great practical importance, the distribution of magnetic induction in soft iron, taking into account the variation of the permeatility with the force. But these again will be left to those who need the results.

\section{Note on Boundary Conditions.}

It may be conveuient to the reader if we bring together certain well-known facts concerning boundary conditions.

Let us regard $\mathrm{V}$ simply as a function of position, not necessarily satisfying $\nabla^{2} V=0$ or any other equation; and, as always, let contours be drawn at small intervals of $V$ each equal to $k$. Then the first space-rate of $\mathrm{V}$ in any direction at a point is inversely as the intercept cut off from a line in that direction by two contours of $\mathrm{V}$ one on each side of the point, and is directly as $K$. Suppose, further, that the whole distribution of $V$ can be represented by a single graph.

1. If we have to make $V$ such that the magnitude and direction of its maximum first space-rate, the Hamiltonian vector $\nabla V$, satisfies given values over a boundary of a given shape. Then it is easy to set off the ends of the contours of $\mathrm{V}$ with a ruler and scale, for their directions are known and also the distance apart of successive pairs. 
2. If we are not given $\nabla \mathrm{V}$ over the boundary but only the first space-rate of $V$ in a given direction. Then there are an indefinite number of ways in which the contours of $V$ may cut the boundary; and as it will not generally be possible to say which of these is consistent with the internal conditions, they must be drawn and modified freehand as the approximation to the internal conditions proceeds. This is usually not difficult.

3. To nake $\mathrm{V}$ continuous at any surface cutting the distribution, all that is necessary is that the ends of the contours of $V$ approaching from the two sides should meet one another at this surface. Whether they meet at an angle or not does not matter.

4. To make the first space-rates of $\mathrm{V}$ in every direction continuous at any surface where $\mathrm{V}$ is continuous, not only must the contours of $\mathrm{V}$ meet one another, but they must pass smoothly into one another without making an angle. For if they made an angle and a straight line were drawn tangent to one branch of the contours at the angle, then the ratio of successive intercepts of this line by the contours of $\mathrm{V}$ would not become unity when the contours were drawn at indefinitely small intervals of $\mathrm{V}$, so that the second space-rate along this straight would be indefinite at the angle.

5. Suppose next that a non-divergent vector is normal to the surfaces $V=$ const., and that the magnitude of the vector is equal to the space-rate of $V$ along the said normal, multiplied by a scalar function of position ; which according to the particular application will be the conductivity, permeability or some other specific constant. Then we may require the conditions which must hold at a boundary where the specific constant has a discontinuity while $\mathrm{V}$ is continuous-as, for example, where magnetic flux passes from air into mild steel. These conditions, which I take from Prof. J. J. Thomson's 'Elements of Electricity and Magnetism,' may be stated thus:-If $K_{1}, K_{2}$ are the aforesaid specific constants on the two sides of the boundary, and $\theta_{1}$ and $\theta_{2}$ are the corresponding angles which the direction of the vector makes 
with the bounding surface, then

$$
\frac{1}{\mathrm{~K}_{1}} \tan \theta_{1}=\frac{1}{\overline{\mathrm{K}}_{2}} \tan \theta_{2} \text {. }
$$

Now if the graph be drawn on a surface wlich is normal to the guiding lines, the direction of the vector lies in the graph, and $\theta_{1}$ and $\theta_{2}$ are the actual angles which one sees. The same is true of the Mercator's map of a spherical surface distribution, since the angles are unchanged by projection. But with our method for screw symmetry, the angles $\theta_{1}$ and $\theta_{2}$ do not immediately appear, and comparison must be made with the angles of the slant sections of the rectangular tubes given on the standard chart.

\section{Miscellaneous Notes on Draughtsmanstip.}

(a) Since with the exception of given boundaries and lines deduced from symmetry no part of the field can be said to be correct until the whole field is correct, it is advisable to begin by covering the whole field with intersecting lines, however erroneous they may be, and then to carry out amendments over wide areas at one time.

(b) In the final stages of a drawing intended improvements often overshoot the mark or cause unforeseen disturbances in the surrounding chequers. It seems well, therefore, to lay aside the indiarubber after a certain accuracy has been reached, and, placing a sheet of tracing-paper over the rough diagram, to draw the intended improvements upon this. And so with all later stages. The tracing-paper diagrams are then compared with one another and the best selected.

(c) The graphic addition of two scalar functions of position is conveniently performed in the way described by Maxwell ("Elementary Treatise on Electricity") by laying the contours drawn on a sheet of tracing-paper at equal intervals of the one over those of the other, covering the two with a clean sheet of tracing-paper, and drawing the diagonals of the chequers formed by the intersecting contours. 


\section{Estimation of Errors.}

To one reading an account of this freehand method without. having worked an example, it might seem as if there were no way of setting a limit to the errors of any particular graph. This, if it were true, would be a serious fault. But, happily, it is not so: for it is commonly necessary to malie several drawings and then select the best of them : so that by the time the draughtsman has reached a drawing which he can scarcely improve upon, he has before him deriations from it in divers directions. The difference, then, between the selected graph and the second best graphs is a measure of the errors of the latter and an outside limit to the errors of the former. The actual errors of the selected graph will be less than this limit, and may be estimated by comparing the errors in the shape of the individual chequers in the best and second best grapls, and taking a fraction, thus :-

$\frac{\text { individual chequer error in best graph }}{\text { same in second best graph }}\left(\begin{array}{c}\text { difference between best and } \\ \text { second best graplis }\end{array}\right)$.

This is the true measure of the errors of the best graph. It depends, of course, on a general mental estimate or appreciation, and is consequently not susceptible of exact definition. But this does not much matter, for if the value of an error be known within two times either way it is usually sufficient.

The difference between the best and second best graphs is less dependent on a mental estimate, and consequently sets a firmer limit to the possible error.

Taking, for example, the graph of the field round a helical line source given in section III. $c$, and laying over it the tracing of the unpublished second-best graph, one sees that the difference in position of the lines in the two graphs nowhere exceeds $\frac{1}{2}$ the linear dimension of the chequer, at the point and in the direction considered. Now I should estimate that the error of the shape of individual chequers in the published graph averaged $\frac{1}{2}$ of the same quantity in the other ; so that $\frac{1}{4}$ of the linear dimensions of the chequer may be taken as the error of position of the lines in the published graph. Now the graph exhibits ten tubes of flow; so that $\frac{1}{4}$ of one 


\section{FREEHAND WAY OF DETERMINIYG STREAM LINES.}

tulbe is $2 \frac{1}{2}$ per cent of the range. This is in the worst parts of the field. Elsewhere the error will be less, but it may still be expected to exceed the errors found when the graph is drawn on a surface normal to the guiding lines, because in the case of screw symmetry we have the added difficulty that the shape of the chequers depend upon its orientation.

\section{Discussion.}

M[1. A. Russfel said that electricians had often to draw lines of force freehand. As a rule they had little more than their intuition to guide them, as the values of many of the variables were only known roughly and the bounding-curves were not simple geometrical figures. They would therefore welcome the paper, as it would enable them to improve their diagrams. The problem of the flow in two-dimensional space could in many cases be satisfactorily solved by experiment-for instance, by Prof. Hele Shar's method. The author had shown, however, that the graphical method could be applied usefully to certain classes of problems in three dimensions which it would be extremely difficult to attack by any other method. The speaker had found difficulty in determining the "chequer ratio" in electrical problems where the extent of the field was tunliwited.

MIr. A. CANPBELL aslied if the author's method would be applicable to a helix of finite leng'th carrying a current.

The Chatruas remarlied that in the method described much lepended on the eye, judgment, and patience.

The Author, in reply to Mr. Campbell, said his method would not apply to the case of a helix of infinite lengtl. 


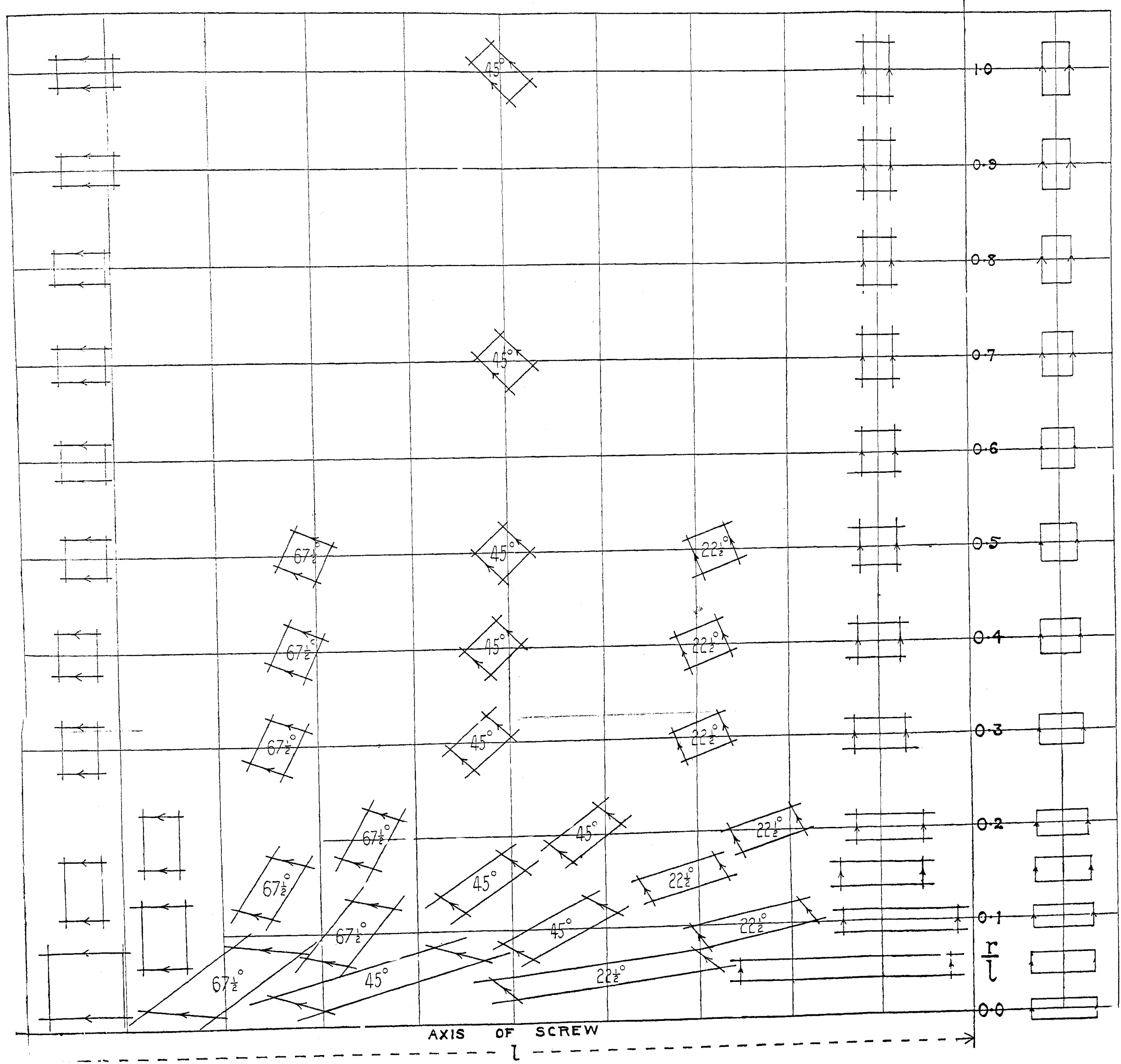

